Supplement of Clim. Past Discuss., 11, 3853-3895, 2015

http://www.clim-past-discuss.net/11/3853/2015/

doi:10.5194/cpd-11-3853-2015-supplement

(C) Author(s) 2015. CC Attribution 3.0 License.

(c) (i)

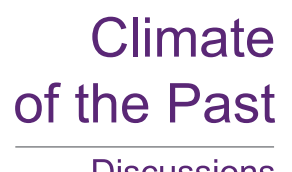

Discussions

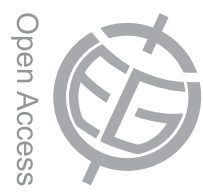

Supplement of

\title{
The influence of non-stationary ENSO teleconnections on reconstructions of paleoclimate using a pseudoproxy framework
}

R. Batehup et al.

Correspondence to: S. McGregor (shayne.mcgregor@unsw.edu.au)

The copyright of individual parts of the supplement might differ from the CC-BY 3.0 licence. 


\section{Supplementary Material}
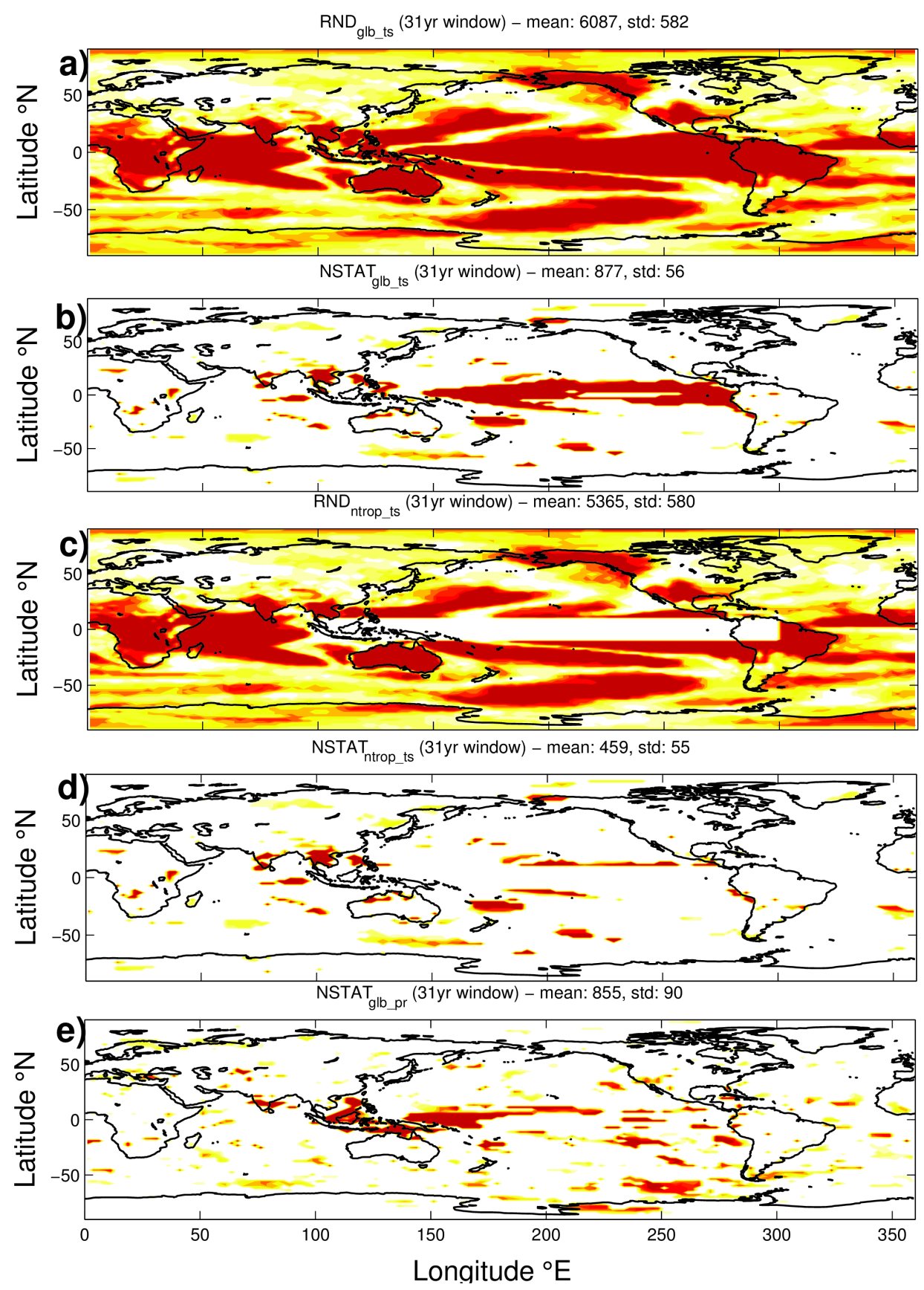

Figure S1. This is a density map of the locations of the possible pseudoproxies for each calibration window for different experiments. This map shows the variation in pseudoproxy selection criteria across calibration windows, and reflects some of the patterns in Figure 2a, as well as showing the physical effect of the experiment criteria. 


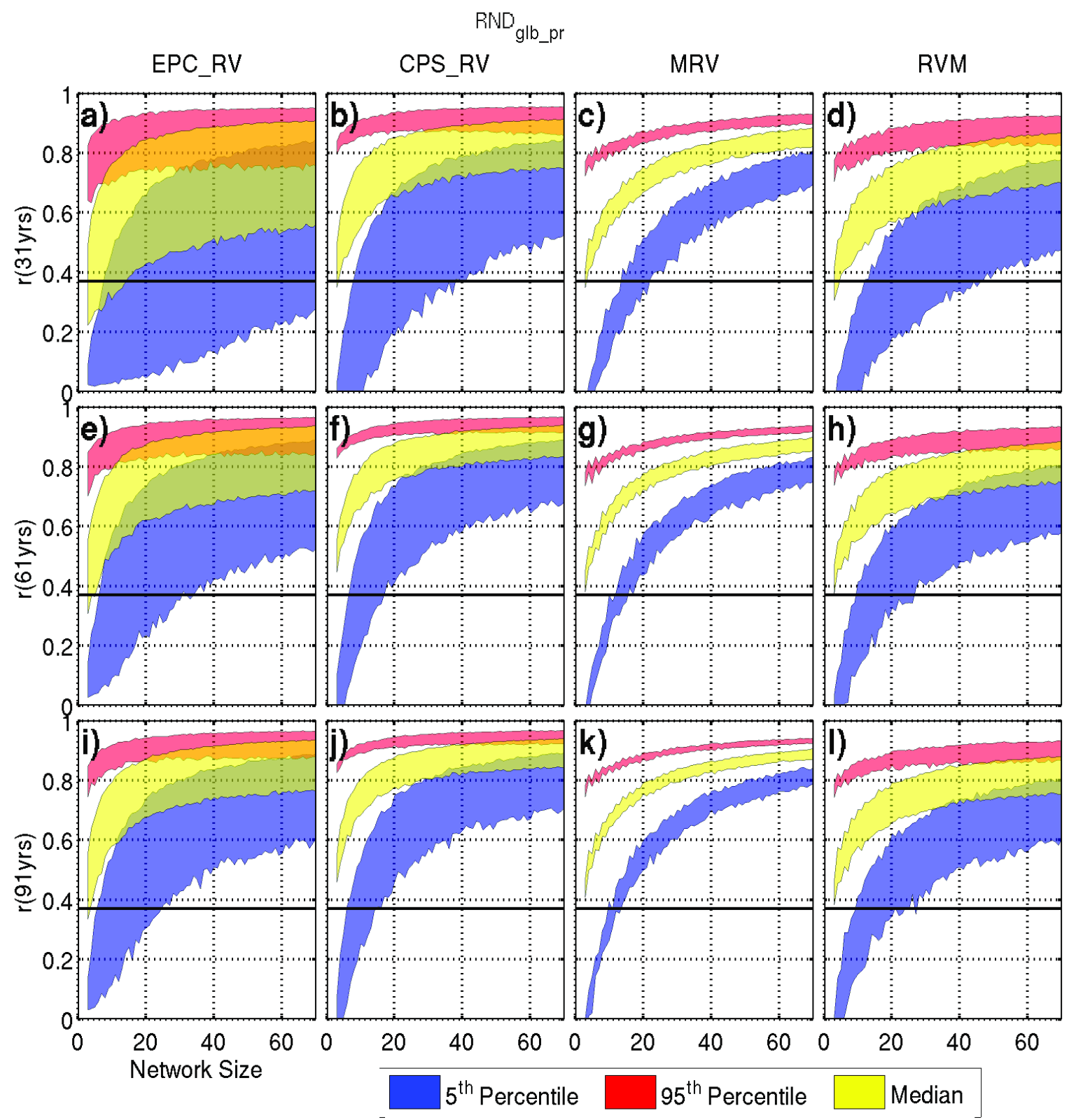

Figure S2. This is the correlation of reconstructions to the Nino3.4 index, and thus is a measure of how skilful the reconstruction method is for the precipitation counterpart of the globally random experiment. This is the same type of plot as Figures 4-7, and the following description is from those figures. The shaded range is the result of using ten different calibration windows within the 499 years of data, and is simply the range of these reconstructions' percentiles. Each column uses one reconstruction method, titled at the top, while each row belongs to calibration windows of a certain size $(31,61$ or 91 years). The three shaded areas are ranges of different percentiles, the 5th, 50th and the 95th percentile. This plot is the same as showing the percentiles of the histogram of reconstruction skill for each of ten calibration windows (and for each group size), but showing the range instead of the ten percentile lines. 


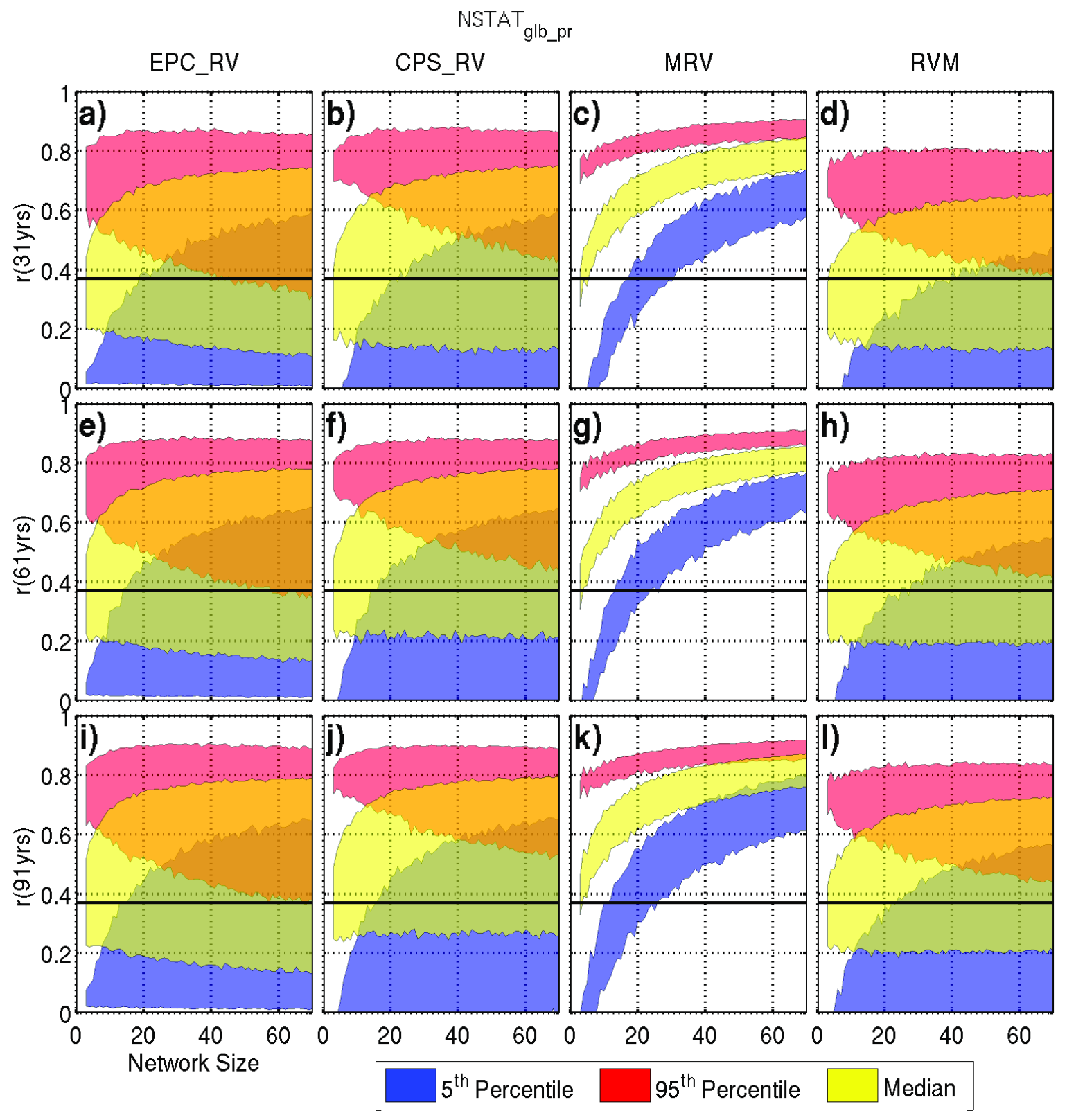

Figure S3. The 5th (blue), 50th (yellow) and 95th (red) percentiles of correlation coefficients calculated between the precipitation based $N S T A T_{g l b}$ experiment pseudo-reconstructions running variance and ENSO running variance (y-axis) plotted against the proxy network size (x-axis). The shaded range is the result of using ten different calibration windows within the 499 years of data, and is simply the range of these reconstructions' percentiles. Each column represents one reconstruction method (titled at the top of each column) while each row represents the length of the calibration windows (titled on the y-axis of each row). 


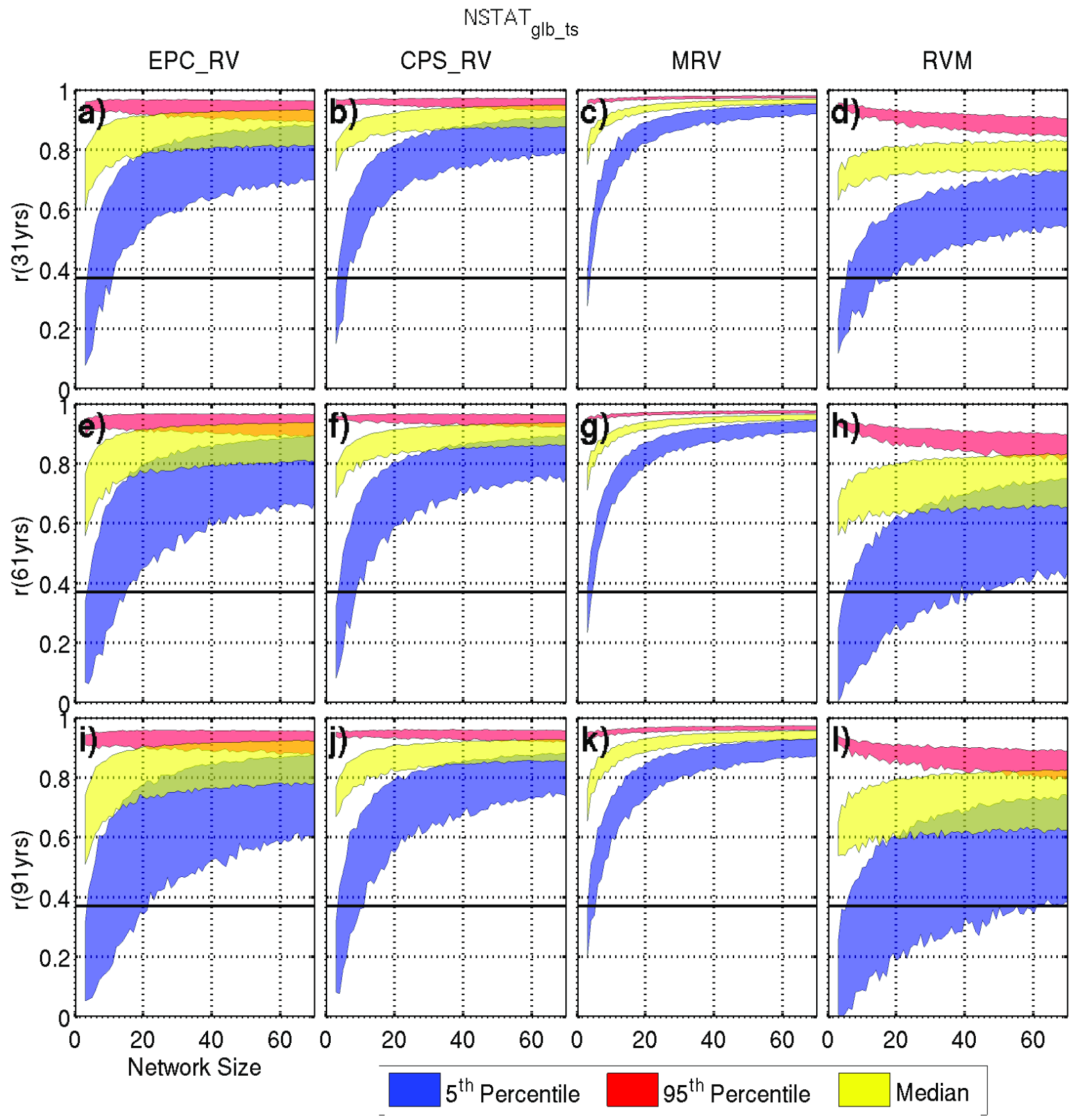

Figure S4. The 5th (blue), 50th (yellow) and 95th (red) percentiles of correlation coefficients calculated between the TS based NSTAT $T_{g l b}$ experiment pseudo-reconstructions running variance and ENSO running variance (yaxis) plotted against the proxy network size (x-axis). The shaded range is the result of using ten different calibration windows within the 499 years of data, and is simply the range of these reconstructions' percentiles. Each column represents one reconstruction method (titled at the top of each column) while each row represents the length of the calibration windows (titled on the y-axis of each row). This figure is similar to Figure 4, but with with the non-stationary pseudoproxy criteria. 


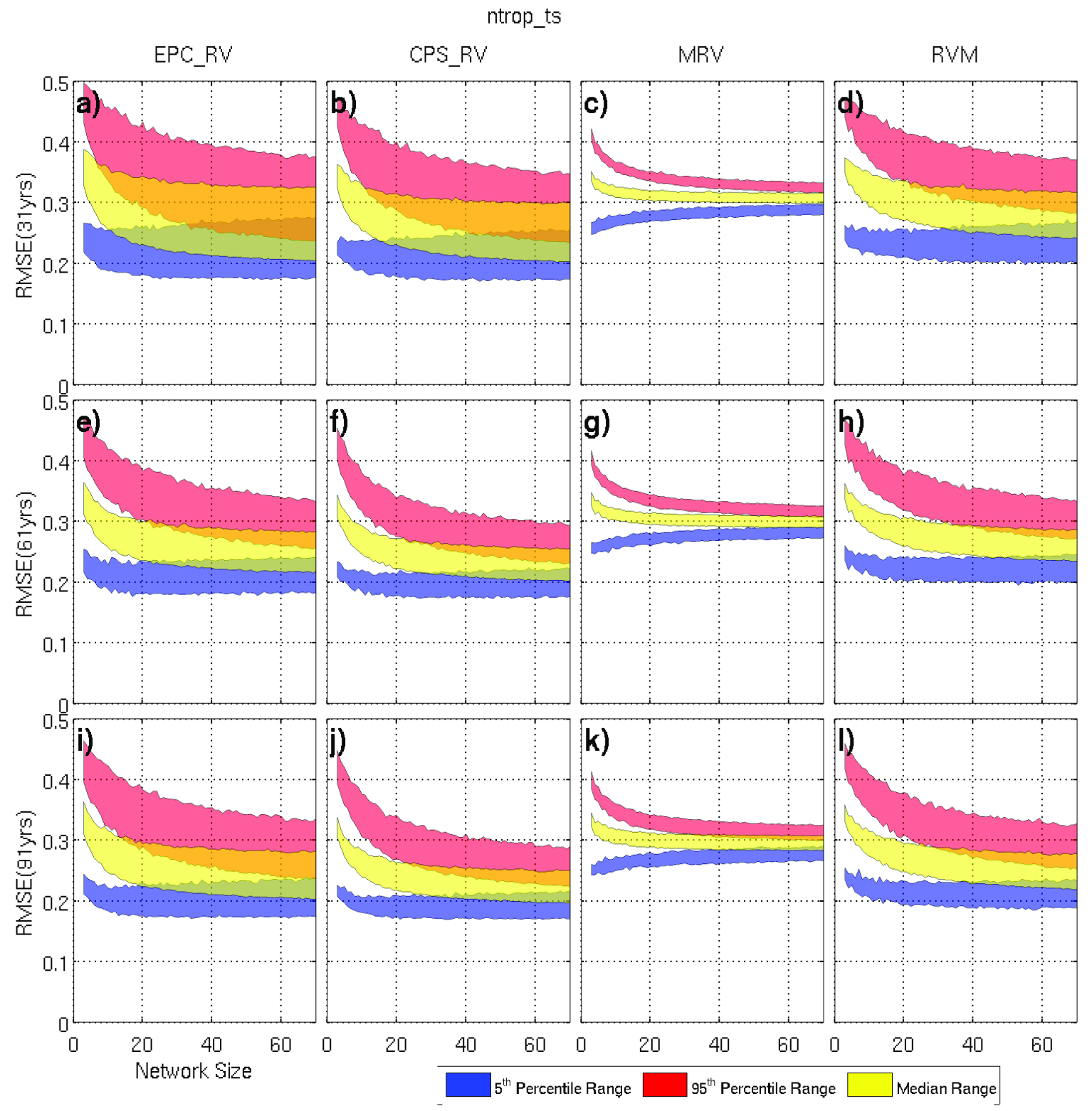

Figure S5. The 5th (blue), 50th (yellow) and 95th (red) percentiles of the root-mean-squared error statistic calculated between the TS based $R N D_{n t r o p}$ pseudoproxy reconstructions running variance and ENSO running variance (y-axis) plotted against the proxy network size (x-axis). The shaded range is the result of using ten different calibration windows within the 499 years of data, and is simply the range of these reconstructions' percentiles. Each column represents one reconstruction method (titled at the top of each column) while each row represents the length of the calibration windows (titled on the y-axis of each row). It is similar to Figures 4-7, except that this shows RMSE instead of correlation for the $R N D_{g l b}$ experiment. 
trop_ts_nstat
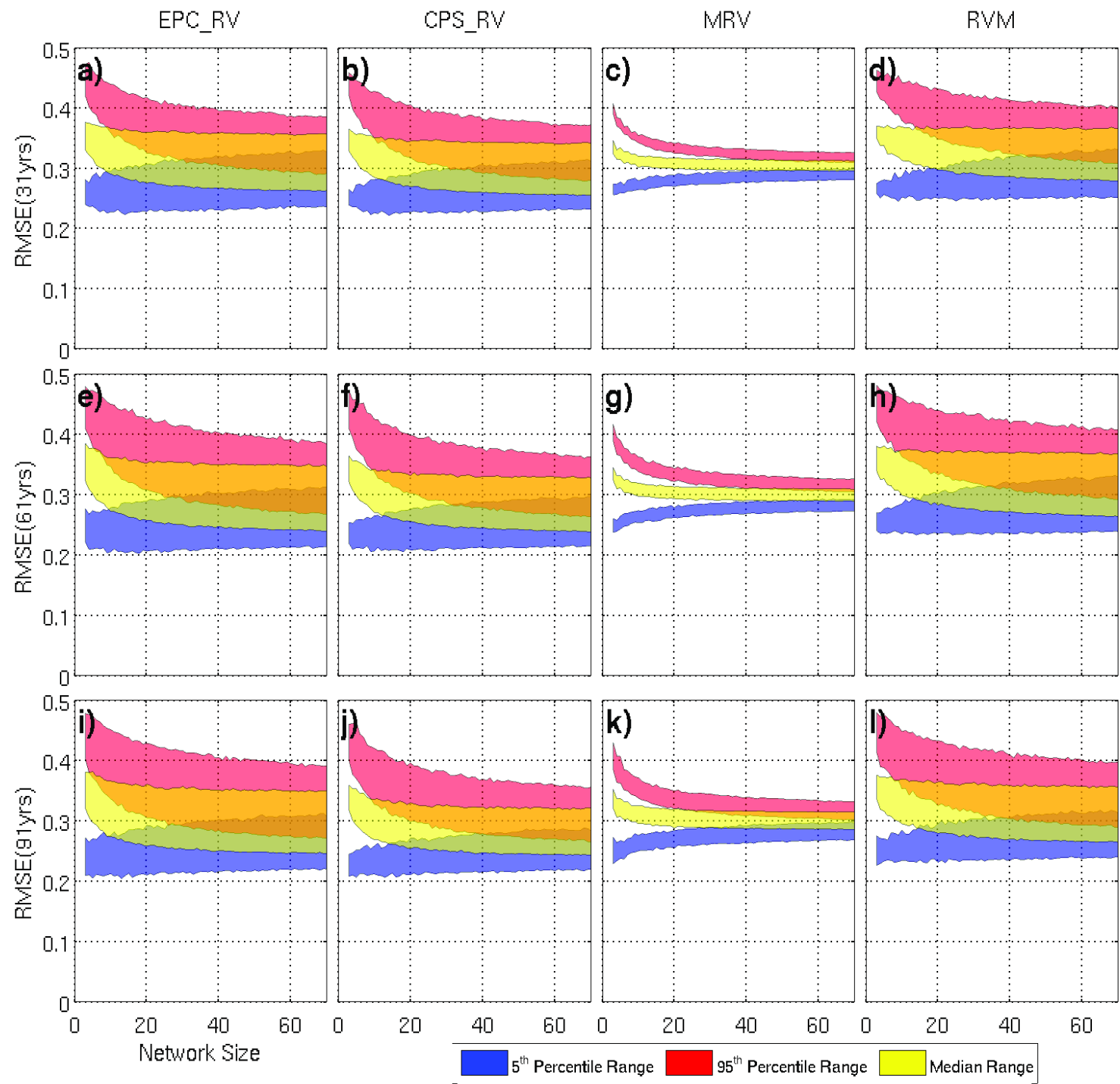

Figure S6. The 5th (blue), 50th (yellow) and 95th (red) percentiles of the root-mean-squared error statistic calculated between the TS based NST AT ntrop pseudoproxy reconstructions running variance and ENSO running variance (y-axis) plotted against the proxy network size (x-axis). The shaded range is the result of using ten different calibration windows within the 499 years of data, and is simply the range of these reconstructions' percentiles. Each column represents one reconstruction method (titled at the top of each column) while each row represents the length of the calibration windows (titled on the y-axis of each row). It is similar to Figures 4-7, except that this shows RMSE instead of correlation for the $N S T A T_{n t r o p}$ experiment. 

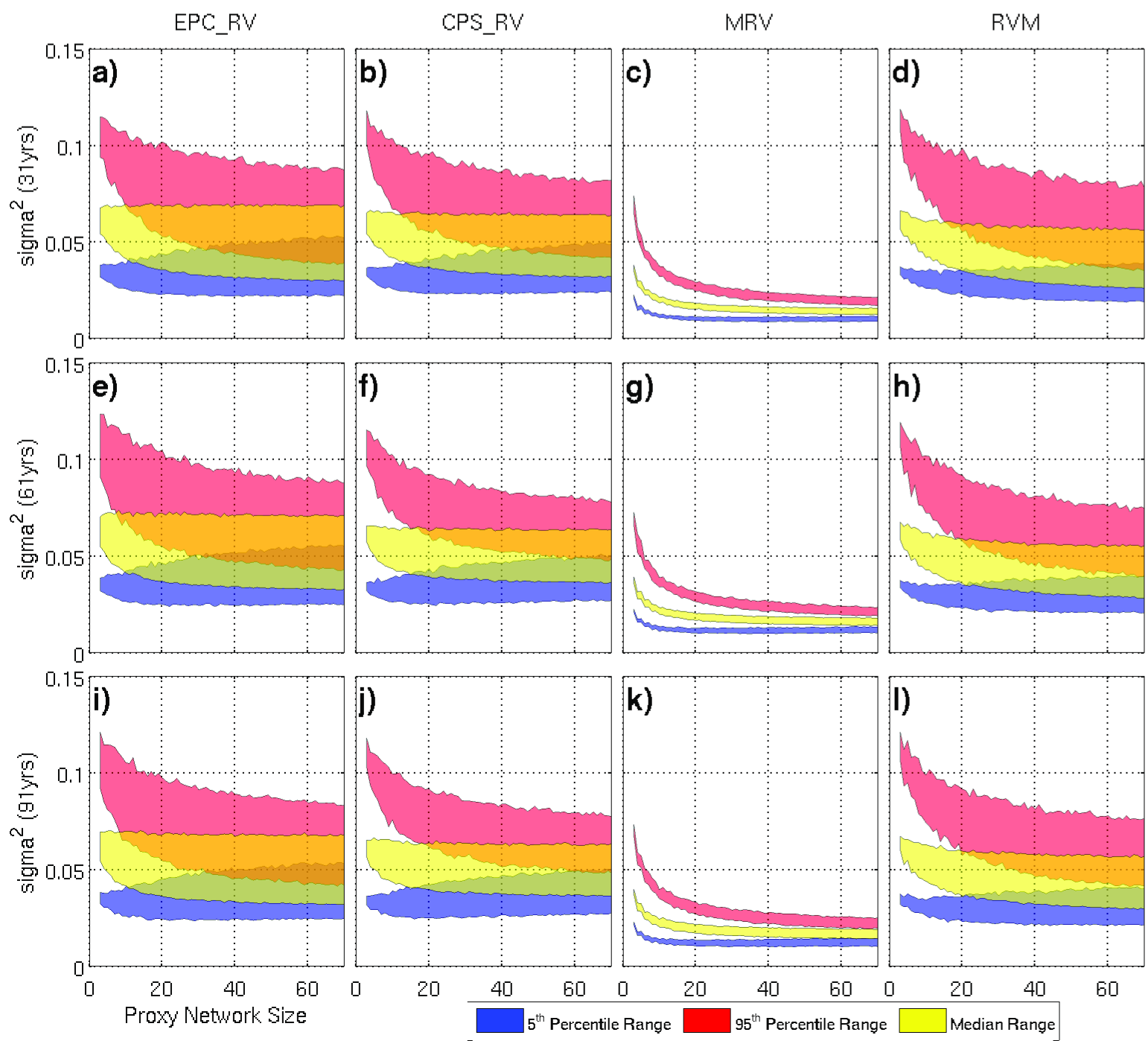

Figure S7. The 5th (blue), 50th (yellow) and 95th (red) percentiles of the variance of the TS based $R N D_{g l b}$ pseudoproxy reconstructions running variance (y-axis) plotted against the proxy network size (x-axis). The shaded range is the result of using ten different calibration windows within the 499 years of data, and is simply the range of these reconstructions' percentiles. Each column represents one reconstruction method (titled at the top of each column) while each row represents the length of the calibration windows (titled on the y-axis of each row). It is similar to Figures 4-7, except that this shows variance instead of correlation for the $R N D_{g l b}$ experiment. 